\title{
Patterns of Brain Injury in Newborns Treated with Extracorporeal Membrane Oxygenation
}

\author{
(D) M.A. Wien, (DM.T. Whitehead, (DD. Bulas, D M. Ridore, (D) Melbourne, (D) G. Oldenburg, (D) B.L. Short, and (D)A.N. Massaro
}

\begin{abstract}
BACKGROUND AND PURPOSE: Neonates treated with extracorporeal membrane oxygenation are at risk for brain injury and subsequent neurodevelopmental compromise. Advances in MR imaging and improved accessibility have led to the increased use of routine MR imaging after extracorporeal membrane oxygenation. Our objective was to describe the frequency and patterns of extracorporeal membrane oxygenation-related brain injury based on MR imaging findings in a large contemporary cohort of neonates treated with extracorporeal membrane oxygenation.
\end{abstract}

MATERIALS AND METHODS: This was a retrospective study of neonatal patients treated with extracorporeal membrane oxygenation from 2005-2015 who underwent MR imaging before discharge. MR imaging and ultrasound studies were reviewed for location and type of parenchymal injury, ventricular abnormalities, and increased subarachnoid spaces. Parenchymal injury frequencies between patients treated with venoarterial and venovenous extracorporeal membrane oxygenation were compared by $\chi^{2}$ tests.

RESULTS: Of 81 neonates studied, $46 \%$ demonstrated parenchymal injury; $6 \%$ showed infarction, mostly in vascular territories (5\% anterior cerebral artery, $5 \%$ MCA, $1 \%$ posterior cerebral artery); and $20 \%$ had hemorrhagic lesions. The highest frequency of injury occurred in the frontal (right, 24\%; left, 25\%) and temporoparietal (right, 14\%; left, 19\%) white matter. Sonography had low sensitivity for these lesions. Other MR imaging findings included volume loss (35\%), increased subarachnoid spaces (44\%), and ventriculomegaly (17\% mild, $5 \%$ moderate, $1 \%$ severe). There were more parenchymal injuries in neonates treated with venoarterial (49\%) versus venovenous extracorporeal membrane oxygenation $(29 \%, P=.13)$, but the pattern of injury was consistent between both modes.

CONCLUSIONS: MR imaging identifies brain injury in nearly half of neonates after treatment with extracorporeal membrane oxygenation. The frontal and temporoparietal white matter are most commonly affected, without statistically significant laterality. This pattern of injury is similar between venovenous and venoarterial extracorporeal membrane oxygenation, though the frequency of injury may be higher after venoarterial extracorporeal membrane oxygenation.

ABBREVIATIONS: $C U S=$ cranial ultrasound; $E C M O=$ extracorporeal membrane oxygenation; $\mathrm{VA}=$ venoarterial; $\mathrm{VV}=$ venovenous

$\mathbf{E}^{x}$ xtracorporeal membrane oxygenation (ECMO) is a therapy for neonates with severe cardiac and/or respiratory failure caused by conditions such as congenital diaphragmatic hernia, persistent pulmonary hypertension, meconium aspiration syn-

Received October 2, 2016; accepted December 5.

From the Divisions of Diagnostic Imaging and Radiology (M.A.W., M.T.W., D.B.) and Neonatology (D.B., M.R., L.M., B.L.S., A.N.M.) and the ECMO Program (G.O.), Children's National Health System, Washington, DC; and The George Washington University School of Medicine (M.T.W., D.B., L.M., A.N.M.), Washington, DC.

This study was supported by the Extracorporeal Life Support Organization.

Please address correspondence to An N. Massaro, MD, Division of Neonatology, The George Washington University School of Medicine, Children's

National Medical Center, 111 Michigan Ave NW, Washington, DC 20010; e-mail: anguyenm@childrensnational.org

http://dx.doi.org/10.3174/ajnr.A5092 drome, and sepsis. Intracranial injury is a major complication and cause of mortality from treatment with ECMO. ECMO-related brain injury can be attributed to hypoxic-ischemic insults caused by cardiopulmonary instability in the pre-ECMO period, sequelae of carotid artery ligation, and hemorrhagic injury related to systemic anticoagulation. ${ }^{1-3}$ The incidence of brain injury after ECMO varies in the literature, but has been estimated to range between $10 \%-59 \% .{ }^{4}$ However, these estimates are limited because of the variability in neuroimaging modalities used to identify injuries, the small sample sizes included in most reports, and the description of historical cohorts who underwent care decades ago.

Initial studies focusing on the documentation and description of neurologic injury from ECMO focused mainly on cranial ultrasound (CUS). More recently, the added utility of head CT over 

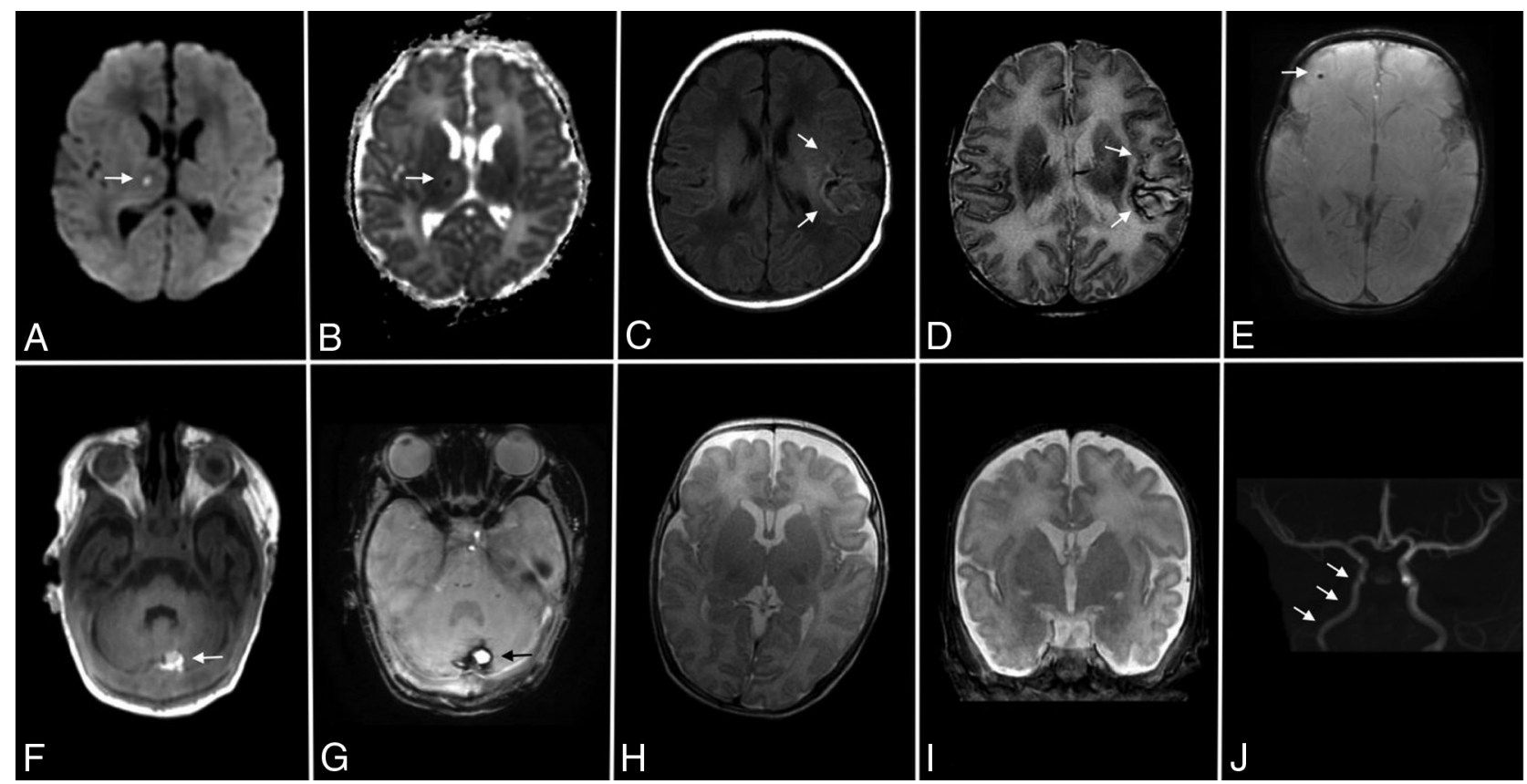

FIG 1. Types of intracranial injury associated with ECMO. Acute infarct within the right thalamus on DWI (arrow, $A$ ) and $A D C$ (arrow, $B$ ) is classified as minor. Subacute infarct within the left MCA territory on T1 (arrows, C) and T2 (arrows, D) is classified as major. Punctate hemorrhage within the right frontal subcortical white matter on T2*-weighted angiography (arrow, $E$ ) is classified as minor. Hemorrhage within the cerebellum on $\mathrm{Tl}$ (arrow, $F$ ) and T2*-weighted angiography (arrow, G) is classified as major. Enlarged subarachnoid spaces are visible on axial T2 $(H)$ and coronal T2 (I) views. Narrowing of the right ICA is visible on TOF MRA (arrows, J).

CUS for identifying brain injury in neonates treated with ECMO has been reported. ${ }^{5}$ Although advances in MR imaging and improved accessibility have led to increased use of routine posttreatment MR imaging, few studies have described patterns of post-ECMO brain injury on MR imaging. Furthermore, most studies have imaged a relatively small cohort of patients ${ }^{4,6-11}$ and do not reflect the current era of care, when time changes and advances have been made with regard to equipment (eg, centrifugal pump, hollow-fiber oxygenator, and cerebral oximetry) and management strategies (eg, approach to anticoagulation) used to provide ECMO life support. Current estimates of the amount of post-ECMO cerebral injury are essential for counseling families regarding the riskbenefit ratio of this life-saving therapy.

The main objective of our study was to describe the frequency and patterns of ECMO-related brain injury based on MR imaging in a relatively large contemporary cohort of neonates treated with ECMO. Secondarily, we aimed to compare the sensitivities of MR imaging and CUS for post-ECMO brain injury and to evaluate whether the type and frequency of brain injury differ between patients undergoing venoarterial (VA) ECMO and those treated with venovenous (VV) ECMO.

\section{MATERIALS AND METHODS}

\section{Study Population and Data Collection}

This institutional review board-approved, Health Insurance Portability and Accountability Act compliant retrospective study included neonatal patients treated with ECMO, admitted to a single-center level IV neonatal intensive care unit in a free-standing academic children's hospital, between July 2005 and February 2015, who underwent brain MR imaging before discharge ac- cording to unit protocol. At our institution, all neonatal patients treated with ECMO undergo MR imaging before discharge when they are deemed clinically stable enough to tolerate the procedure. Demographic and clinical data were compiled from the Extracorporeal Life Support Organization registry and medical records including demographic and presenting characteristics as well as mode and duration of ECMO.

\section{Assessment of Brain Injury with MR Imaging}

MRIs were reviewed for location and type of parenchymal injury by using a method similar to that described by Bulas et al. ${ }^{5}$ Infarctions were classified as minor (small $[<1 \mathrm{~cm}]$ foci of signal abnormality [Fig $1 A,-B])$ or major $(>1 \mathrm{~cm}$, including large vascular territories [Fig $1 C,-D])$. Hemorrhages classified as minor included extra-axial hemorrhages without mass effect, parenchymal hemorrhages (single or multiple) smaller than $1 \mathrm{~cm}$ (Fig $1 F)$, and grade 1 or 2 intraventricular hemorrhage. All other hemorrhages were classified as major (Fig 1G). Lesions were also assessed based on their acuity, taking into account the proximity to ECMO decannulation. Specifically, attention was paid to signal characteristics on diffusion, T1, and T2 sequences to classify infarcts and hemorrhages as acute, subacute (early and late in the case of hemorrhage), or chronic by methods previously described. ${ }^{12,13}$ Enlargement of the subarachnoid spaces (Fig $1 H,-I$ ) was assessed by measuring the space anterior to the frontal poles. If greater than or equal to $6 \mathrm{~mm}$, the spaces were labeled "enlarged."

All MR images were performed on either a $1.5 \mathrm{~T}$ or 3T MR scanner (GE Healthcare, Milwaukee, Wisconsin). Scans were performed with a "feed and bundle" protocol during the patients' natural sleep. Sedation was used at the discretion of the clinical provider, typically in cases where the baby was weaning from sed- 
Table 1: Clinical characteristics of patient population ${ }^{\mathrm{a}}$

\begin{tabular}{|c|c|c|c|c|}
\hline Characteristics & $\begin{array}{l}\text { Total } \\
(n=81)\end{array}$ & $\begin{array}{l}\text { VA ECMO } \\
(n=67)\end{array}$ & $\begin{array}{l}\text { VV ECMO } \\
(n=14)\end{array}$ & $\begin{array}{c}P \\
\text { Value }^{b}\end{array}$ \\
\hline Gestational age (wk) & $38.12 \pm 3.12$ & $37.98 \pm 2.82$ & $38.79 \pm 4.35$ & .382 \\
\hline Birth weight (kg) & $3.16 \pm 0.76$ & $3.12 \pm 0.72$ & $3.35 \pm 0.94$ & .303 \\
\hline Male, no. (\%) & $48(59)$ & $39(81)$ & 9 (19) & .674 \\
\hline \multicolumn{5}{|l|}{ Race, no. (\%) } \\
\hline White & $16(20)$ & $13(81)$ & $3(19)$ & .804 \\
\hline Black & $41(50)$ & $35(85)$ & $6(15)$ & \\
\hline Other & $24(30)$ & $19(79)$ & $5(21)$ & \\
\hline \multicolumn{5}{|l|}{ Apgar score ${ }^{c}$} \\
\hline $1 \min ^{d}$ & $5(5)$ & $5(4)$ & $4(5)$ & .699 \\
\hline $5 \min ^{d}$ & $7(3)$ & $7.5(2)$ & $7(3)$ & 691 \\
\hline \multicolumn{5}{|l|}{ Diagnosis, no. (\%) } \\
\hline MAS & $34(42)$ & $25(37)$ & $9(64)$ & .033 \\
\hline $\mathrm{CDH}$ & $17(21)$ & $17(25)$ & $0(0)$ & \\
\hline PPHN & $13(16)$ & $12(18)$ & $1(7)$ & \\
\hline Sepsis & $11(14)$ & $7(10)$ & $4(29)$ & \\
\hline Other & $6(7)$ & $6(9)$ & $0(0)$ & \\
\hline Age on ECMO (d) & $2(2)$ & $2(3)$ & $1.5(1.5)$ & .645 \\
\hline ECMO hours ${ }^{c}$ & 168 (127) & 190 (128) & $103(98)$ & .006 \\
\hline Age at MRI $(d)^{c}$ & $26(24)$ & $32(24)$ & $16(10)$ & .560 \\
\hline $\begin{array}{l}\text { Time from decannulation } \\
\text { to MRI (d) }\end{array}$ & $12(16)$ & $13(19)$ & $8(4)$ & .034 \\
\hline
\end{tabular}

Note:- $\mathrm{CDH}$ indicates congenital diaphragmatic hernia; MAS, meconium aspiration syndrome; PPHN, persistent pulmonary hypertension of the newborn.

${ }^{a}$ Data presented as mean \pm SD or proportions except where noted.

${ }^{\mathrm{b}}$ Comparison between VA and VV.

'Data presented as median (interquartile range)

${ }^{\mathrm{d}}$ Data available for $79 / 81$ patients.

e Data available for $67 / 81$ patients.

atives or failed a prior nonsedated examination. Technical parameters varied throughout the 10 -year imaging period, though most scans included $\mathrm{T} 1$ fast-spoiled gradient-echo, T2, proton density, DWI, susceptibility-weighted angiography, or T2* gradient-echo sequences. Spectroscopy was performed in some neonates, all with a $2 \times 2 \mathrm{~cm}$ voxel placed in the left basal ganglia. The TE varied from intermediate $(144 \mathrm{~ms})$ in most patients to short (35 $\mathrm{ms}$ ) and long (270 ms or $288 \mathrm{~ms}$ ) in others. Some patients also underwent arterial spin-labeling perfusion imaging as part of a newer protocol. All MR imaging examinations were also assessed for evidence of a right distal ICA flow void of asymmetrically decreased caliber (narrowing [Fig $1 J]$ ), a common, albeit nonspecific, finding suggesting carotid stenosis, occlusion, or slow flow in patients who have undergone right carotid decannulation after VA ECMO.

Images were reviewed on a PACS workstation by a boardcertified neuroradiologist with a clinical practice of $100 \%$ pediatric neuroradiology and more than 5 years of clinical experience (M.T.W.) as well as by a pediatric neuroradiology fellow (M.A.W.). Interpretations were determined in consensus. Reviewers were blinded to the clinical data and the clinical radiology reports corresponding to each examination.

\section{Assessment of Brain Injury by CUS}

CUS is routinely performed before ECMO to assess for major abnormalities or injuries that would exclude patients from ECMO, then daily to every other day during ECMO to monitor for hemorrhagic complications that would prompt discontinuation of ECMO. The CUS studies we used were reviewed by a board-certified pediatric radiologist with a clinical practice of $100 \%$ pediatric radiology and more than 25 years of clinical expe- rience (D.B.). Because more than 1 CUS per study patient was typically available for review, the cumulative assessment of imaging was included for analysis. In other words, of serial sonography examinations, those with the most severe findings were used to document injury and compare with subsequent MR imaging. As with MR imaging, these examinations were also reviewed for location and type of parenchymal injury by using a method similar to that described by Bulas et al. ${ }^{5}$

\section{Data Analysis}

Descriptive statistics included standard measures of central tendency/variance and frequencies for continuous and categoric variables, respectively. Parenchymal injury frequencies were mapped by brain region using Matlab software (MathWorks, Natick, Massachusetts). Frequencies of injury between patients treated with VA ECMO and those treated with VV ECMO were compared by $\chi^{2}$ tests.

\section{RESULTS}

\section{Study Population}

One hundred sixty-one neonates underwent ECMO in the period from July 2005 to February 2015. Overall ECMO mortality was $13 \%$. Of 140 total survivors, 81 (58\%) underwent imaging with both CUS and MR imaging. Of the 81 neonates who underwent imaging, mean birth weight was $3.16 \pm 0.76 \mathrm{~kg}$, gestational age at birth was $38.0 \pm 3.12$ weeks, and $59 \%$ were male. The most common diagnosis on admission was meconium aspiration syndrome (42\%), followed by congenital diaphragmatic hernia (21\%), persistent pulmonary hypertension of the neonate (16\%), and sepsis (14\%). Most neonates were started on ECMO within 1 week of birth, most commonly on the second day of life. Median duration of ECMO was 7 days (range, 2-18 days), and median age at MR imaging was 26 days (Table 1 ).

\section{Frequency, Type, and Location of Brain Injury by MR Imaging}

Of the 81 neonates studied, 37 (46\%) demonstrated imaging evidence of intracranial injury. Hemorrhagic lesions were identified in 19 patients $(23 \%)$. Of these, most $(84 \%)$ were classified as minor and $16 \%$ were classified as major. Most hemorrhages demonstrated signal intensities that were not specific for timing in terms of association with ECMO. In other words, these hemorrhages could have occurred either before ECMO, during ECMO, or in the interim between ECMO decannulation and MR imaging. Infarction was seen in 18 patients (22\%), mostly in vascular territories ( $5 \%$ anterior cerebral artery, $5 \% \mathrm{MCA}, 1 \%$ posterior cerebral artery). Of these, $56 \%$ were classified as minor and $44 \%$ were classified as major. Only approximately half of these lesions could 
Table 2: Distribution of hemorrhagic and ischemic brain injuries by $\mathrm{MRI}^{\mathrm{a}}$

\begin{tabular}{lcc}
\hline Description of Injury & $\begin{array}{c}\text { Hemorrhagic } \\
\text { Injury }(\boldsymbol{n}=19)\end{array}$ & $\begin{array}{c}\text { Ischemic } \\
\text { Injury }(\boldsymbol{n}=18)\end{array}$ \\
\hline Focality & $4(21)$ & $4(22)$ \\
Unifocal (total) & $3(16)$ & $3(17)$ \\
Unilateral right & $1(5)$ & $1(6)$ \\
Unilateral left & $15(79)$ & $14(78)$ \\
Multifocal (total) & $1(5)$ & $1(6)$ \\
Unilateral right & $2(10)$ & $4(22)$ \\
Unilateral left & $12(63)$ & $9(50)$ \\
Bilateral & $16(84)$ & $16(89)$ \\
Supratentorial & $6(32)$ & $1(6)$ \\
Infratentorial & & \\
Anatomic location & $2(10)$ & $12(67)$ \\
Cortex and white matter & $10(52)$ & $5(28)$ \\
White matter only & $2(10)$ & $7(39)$ \\
Deep gray nuclei & $3(16)$ & $1(6)$ \\
Brain stem & $5(26)$ & $0(0)$ \\
Cerebellum & & \\
Vascular territory & $\mathrm{NA}$ & $4(22)$ \\
ACA & $\mathrm{NA}$ & $4(22)$ \\
MCA & $\mathrm{NA}$ & $1(6)$ \\
PCA & & \\
Extra-axial & $7(37)$ & $\mathrm{NA}$ \\
Minor (no mass effect) & $1(5)$ & $\mathrm{NA}$ \\
Major (mass effect) & & \\
\hline
\end{tabular}

Note:-ACA indicates anterior cerebral artery; NA, not applicable; PCA, posterior cerebral artery.

${ }^{a}$ Data presented as no. of patients (\%).

be definitively associated with ECMO based on timing from signal intensity. Distribution of hemorrhagic and ischemic injuries are described in Table 2.

Overall, the highest frequency of injury occurred in the frontal (right, 24\%; left, 25\%) and temporoparietal (right, 14\%; left, $19 \%$ ) white matter (Fig $2 A$ ). There was a slight left-sided predominance of injury, but this difference was not statistically significant $(P>.05)$. Other MR imaging findings included volume loss $(35 \%)$, increased subarachnoid spaces $(44 \%)$, and ventriculomegaly ( $17 \%$ mild, $5 \%$ moderate, $1 \%$ severe; Table 3 ).

\section{MR Spectroscopy and Arterial Spin-Labeling}

MR spectroscopy was performed on 25 neonates. Fifteen underwent intermediate TE only (144 ms). Nine underwent short TE (35 ms) and long TE (270 or $288 \mathrm{~ms}$ ). One underwent only long TE (288 ms). Six (24\%) had evidence of lactate. Age at MR imaging was similar between infants with and without lactate (median age, 17 days [interquartile range, 4 days] versus 17 days [interquartile range, 23 days], respectively; $P>.05$ ), as was proximity of scan to day of decannulation (median, 7 days [interquartile range, 16 days] versus 8.5 days [interquartile range, 9 days]; $P>.05)$. Of the 6 infants with lactate who underwent MR spectroscopy, 2 had histories of perinatal asphyxia and 3 had significant postnatal events requiring cardiopulmonary resuscitation. All neonates had normal ratios of $\mathrm{NAA} / \mathrm{Cr}$ and $\mathrm{Cho} / \mathrm{Cr}$ for their ages.

A total of 24 neonates underwent arterial spin-labeling perfusion imaging. Most images were normal $(n=18 ; 75 \%)$. Three infants $(13 \%)$ had symmetrically increased perfusion, and 1 infant had symmetrically decreased perfusion. Asymmetric perfusion (left side greater than right) was observed in 2 infants.

\section{Comparison of MR Imaging and CUS Assessments of Brain Injury}

Compared with MR imaging, CUS had low sensitivity for intraparenchymal lesions (Fig 2B). CUS missed 15 of 16 (94\%) minor hemorrhages and 1 of 3 (33\%) major hemorrhages. The missed major hemorrhage was located in the right parietotemporal cortex and subcortical white matter. CUS missed all minor infarctions and 3 of 8 (38\%) major infarctions. Of the major infarctions missed, all 3 involved MCA territories.

\section{Comparison of Brain Injury in VA ECMO Versus VV ECMO} Most (82\%) patients were treated with VA ECMO. Distribution of diagnoses differed between the 2 groups, with babies with congenital diaphragmatic hernia being exclusively treated with VA ECMO. Patients treated with VA ECMO spent significantly more time on ECMO compared with those treated with VV ECMO (7.9 days versus 4.3 days, respectively; $P=.006$ ). Otherwise, baseline characteristics were similar between the 2 groups (Table 1). Comparison of MR imaging findings based on mode of ECMO is summarized in Table 3. More parenchymal injuries were seen in patients treated with VA ECMO (49\%) compared with those treated with VV ECMO (29\%), though this difference was not statistically significant $(P=.16)$. The pattern of injury was consistent between the 2 modes (Fig 2C,D). All major hemorrhages and major infarcts were seen in patients on VA ECMO. Patients treated with VA ECMO had higher frequency of increased subarachnoid spaces (51\%) compared with those treated with VV ECMO (14\%; $P=.01)$. Right ICA narrowing was present in $73 \%$ of patients $(n=59)$. However, as expected, narrowing was much more common in patients treated with VA ECMO than those treated with VV ECMO (85\% versus $14 \%$; $P<.001)$. Of the patients on VA ECMO who had arterial spin-labeling as part of their scan, most $(14 / 17$ [82\%]) showed symmetric perfusion.

\section{DISCUSSION}

In the largest, most comprehensive study to date, we used MR imaging to describe the frequency and patterns of brain injury in neonates who have undergone therapy with ECMO. Nearly half of surviving neonatal patients treated with ECMO had intracranial injury detected by MR imaging, and these lesions were largely missed by CUS. We describe both structural changes (such as parenchymal hemorrhage and infarction, enlarged subarachnoid spaces, and ventriculomegaly) and physiologic changes (such as differences in cerebral perfusion by arterial spin-labeling and metabolic profiles by MR spectroscopy). There was a higher frequency of injury in patients treated with VA ECMO compared with those treated with VV ECMO, but the differences were not statistically significant. The patterns of injury were similar between the 2 modes of cannulation. These data can serve as important benchmarks that can be used when counseling families about the risks, benefits, and potential outcomes of ECMO support in the modern era of care.

Our findings are overall in concert with, and serve to complement, those of prior studies examining ECMO-associated patterns of intracranial injury on CUS, CT, and MR imaging. The prevalence of injury reported in the current study (46\%) is similar compared with prior CT-based studies performed at our institu- 

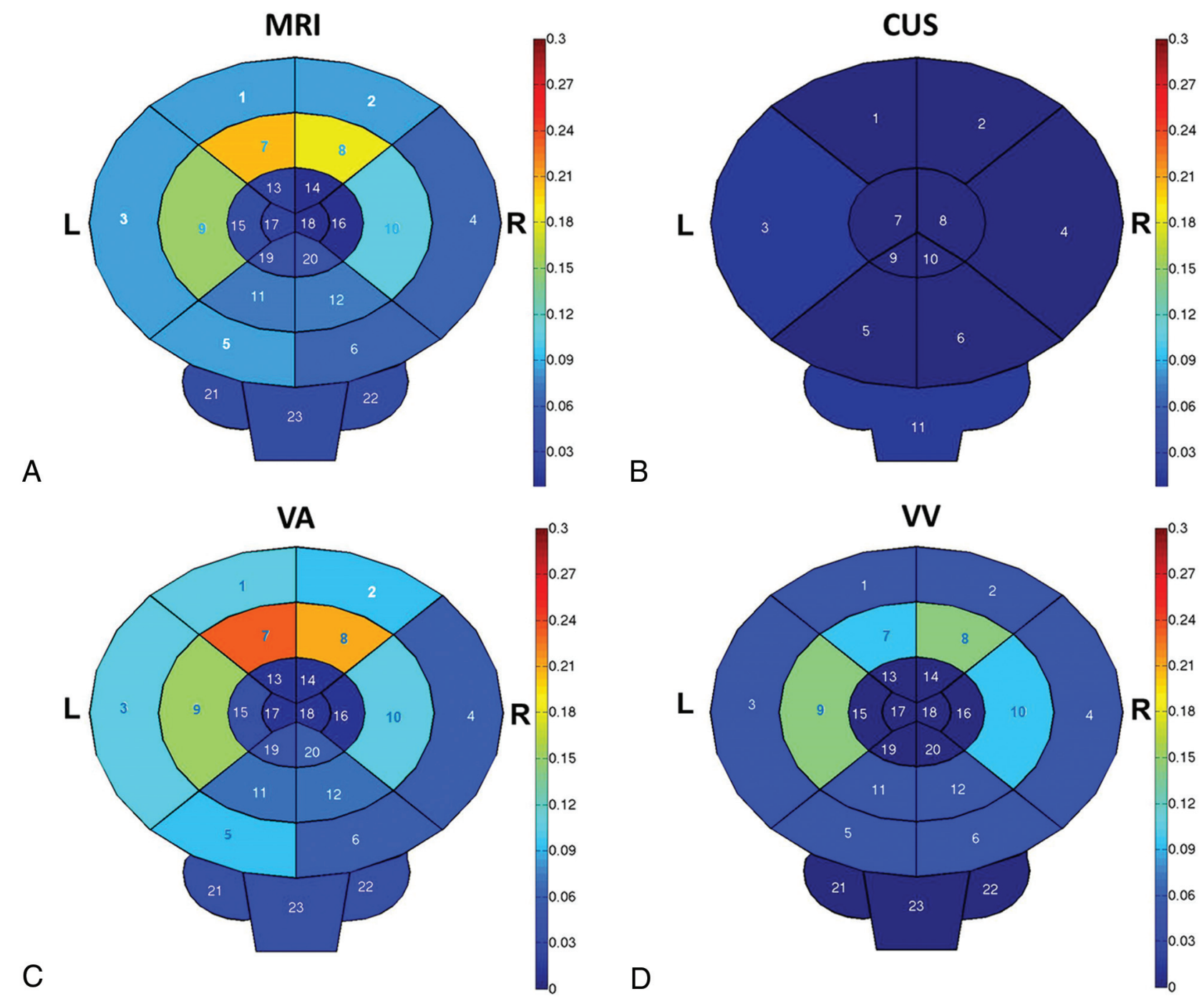

C

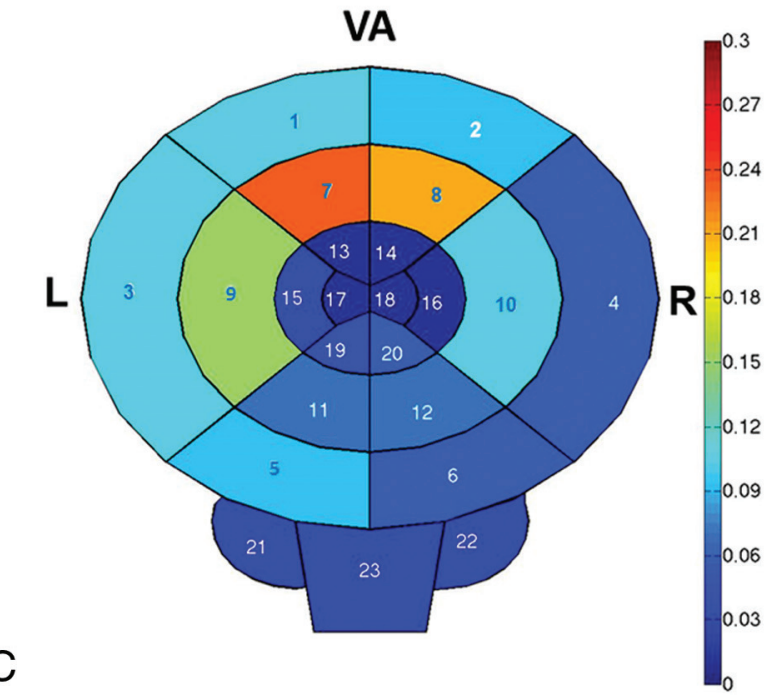

FIG 2. Location and frequency of brain injury. Heat maps demonstrate frequency of parenchymal injury on MR imaging $(A)$ and sonography $(B)$ in addition to comparison between $\mathrm{VA}(C)$ and $\mathrm{VV}(D)$ ECMO based on MR imaging lesions. MR imaging region key: 1, frontal cortex (left); 2 , frontal cortex (right); 3, temporoparietal cortex (left); 4, temporoparietal cortex (right); 5, occipital cortex (left); 6, occipital cortex (right); 7, frontal white matter (left); 8, frontal white matter (right); 9, temporoparietal white matter (left); 10, temporoparietal white matter (right); 11, occipital white matter (left); 12, occipital white matter (right); 13, caudate (left); 14, caudate (right); 15, putamen (left); 16, putamen (right); 17, globus pallidus (left); 18, globus pallidus (right); 19, thalamus (left); 20, thalamus (right); 21, cerebellum (left); 22, cerebellum (right); and 23, brain stem. Sonography region key: 1, frontal (left); 2, frontal (right); 3, temporoparietal (left); 4, temporoparietal (right); 5, occipital (left); 6, occipital (right); 7, basal ganglia (left); 8, basal ganglia (right); 9 , thalamus (left); 10, thalamus (right); and 11, posterior fossa.

tion. ${ }^{5,14}$ Likewise, similar rates of injury have been reported by prior studies evaluating injury on MR imaging. ${ }^{6,9}$ We used a classification scheme previously devised by our group that specifically describes injuries known to occur in patients with ECMO. ${ }^{5}$ It should be noted that studies that did not include volume loss or increased subarachnoid spaces as a form of injury have described lower rates of injury (17\%-26\%). ${ }^{15,16}$ That CUS is less sensitive than MR imaging (or CT) is also consistent with prior studies. ${ }^{5,6,9,14-16}$ Although we used the cumulative assessment of brain injury across serial CUS to optimize the sensitivity of this technique, ${ }^{16}$ it is acknowledged that the direct comparison of sensitivities between CUS and MR imaging is limited because these studies were not performed at the same time. We cannot exclude that injuries occurring between the last CUS and performance of the MR imaging could impact our estimates of injury frequencies.
The most common type of injury involved intraparenchymal ischemic or hemorrhagic lesions, most frequently in the frontal, parietal, and temporal subcortical white matter, without significant laterality. Ischemic lesions or infarctions likely result from a combination of risk factors, including pre-ECMO hemodynamic instability and hypoxia as well as potential cerebral blood flow alterations associated with cannulation. The increased risk of hemorrhage in patients treated with ECMO may relate to treatment with systemic anticoagulation as well as to increased cerebral venous pressure resulting from cannulation/ligation of the right internal jugular vein causing venous outflow obstruction. ${ }^{17}$ The same alteration in venous physiology has been implicated in causing the enlarged subarachnoid spaces in these patients as a result of decreased CSF resorption. ${ }^{18}$ The relatively high frequency of increased subarachnoid spaces in our study (44\%) is overall similar to the prevalence reported in prior studies. ${ }^{5,6}$ 
Table 3: Comparison of intracranial injuries seen on MRI by type of ECMO ${ }^{a}$

\begin{tabular}{|c|c|c|c|c|}
\hline MRI Findings & $\begin{array}{l}\text { Total } \\
(n=81)\end{array}$ & $\begin{array}{l}\text { VA ECMO } \\
(n=67)\end{array}$ & $\begin{array}{l}\text { VV ECMO } \\
(n=14)\end{array}$ & $\begin{array}{c}P \\
\text { Value }^{b}\end{array}$ \\
\hline $\begin{array}{l}\text { Any injury (hemorrhage, infarction, } \\
\text { volume loss, increased } \\
\text { subarachnoid spaces) }\end{array}$ & $37(46)$ & $33(49)$ & $4(29)$ & .157 \\
\hline Any hemorrhage & $19(23)$ & $15(22)$ & $4(26)$ & .247 \\
\hline Major hemorrhage & $3(4)$ & $3(4)$ & $0(0)^{\prime}$ & .420 \\
\hline Minor hemorrhage & $16(20)$ & $12(18)$ & $4(29)$ & .362 \\
\hline Any infarction & $18(22)$ & $16(24)$ & $2(14)$ & .432 \\
\hline $\begin{array}{l}\text { Major infarction (total including } \\
\text { arterial, }{ }^{\mathrm{C}} \text { venous infarcts, } \\
\text { and others) }\end{array}$ & $8(10)$ & $8(12)$ & $0(0)^{\prime}$ & .173 \\
\hline Arterial: ACA territory & $4(5)$ & $4(6)$ & $0(0)$ & .879 \\
\hline Arterial: MCA territory & $4(5)$ & $4(6)$ & $0(0)$ & .879 \\
\hline Arterial: PCA territory & $1(1)$ & $1(1)$ & $0(0)$ & .646 \\
\hline Minor infarctions (total) & $10(13)$ & $8(12)$ & $2(14)$ & .808 \\
\hline Cerebellar injury & $5(6)$ & $5(7)$ & $0(0)$ & .291 \\
\hline \multicolumn{5}{|l|}{ Volume loss } \\
\hline Any & $28(35)$ & $25(37)$ & $3(21)$ & .256 \\
\hline Cortical & $5(6)$ & $5(7)$ & $0(0)$ & .291 \\
\hline White matter & $27(33)$ & $24(36)$ & $3(21)$ & .299 \\
\hline Deep gray nuclei & $3(4)$ & $3(4)$ & $0(0)$ & .420 \\
\hline Cerebellum & $3(4)$ & $3(4)$ & $0(0)$ & .420 \\
\hline Brain stem & $3(4)$ & $3(4)$ & $0(0)$ & .420 \\
\hline \multicolumn{5}{|l|}{ Ventricular size } \\
\hline Normal & $60(74)$ & $49(73)$ & $11(79)$ & .673 \\
\hline Slit & $2(3)$ & $2(3)$ & $0(0)^{\prime}$ & .513 \\
\hline Mild ventriculomegaly & 14 (17) & $11(16)$ & $3(21)$ & .652 \\
\hline Moderate ventriculomegaly & $4(5)$ & $4(6)$ & $0(0)^{\prime}$ & .879 \\
\hline Severe ventriculomegaly & $1(1)$ & $1(1)$ & $0(0)$ & .646 \\
\hline Increased subarachnoid spaces & $36(44)$ & $34(51)$ & $2(14)$ & .012 \\
\hline
\end{tabular}

${ }^{a}$ Data presented as no. of patients (\%).

${ }^{\mathrm{b}}$ Comparison between VA and VV.

cThere was overlap among arterial territories on some studies.

Of the neonates who had MR spectroscopy of the left basal ganglia included as part of their MR imaging protocol, 24\% demonstrated evidence of anaerobic metabolism with the presence of lactate. $\mathrm{NAA} / \mathrm{Cr}$ and $\mathrm{Cho} / \mathrm{Cr}$ ratios were normal in all patients, suggesting retained neuronal/cell membrane integrity within the areas of interest. Only 1 prior study evaluated MR spectroscopy in 9 neonates who had undergone ECMO and reported similar results regarding normal NAA/Cr and Cho/Cr ratios, though only 1 of their patients had mildly elevated lactate. ${ }^{10}$ It is important to note that observation of lactate may be dependent upon the temporal proximity of imaging to the precipitating hypoxic-ischemic event. Because the presence of lactate on MR spectroscopy has been associated with poor outcomes in neonates with hypoxicischemic encephalopathy and in other high-risk populations, ${ }^{19,20}$ evaluating infants with MR spectroscopy after ECMO may allow for identification of a subset of infants with the most significant brain injury.

Similarly, although most neonates had normal perfusion by arterial spin-labeling, 3 (13\%) had symmetrically increased perfusion, which can be seen as a postischemic reperfusion response. ${ }^{21}$ It is uncertain why most patients treated with VA ECMO had normal, symmetric cerebral blood flow. This finding may relate to compensation by collateralization, such as from the vertebrobasilar system. Both of the neonates with asymmetric perfusion in our study demonstrated left side greater than right. It is possible that these infants had compromise to their right hemispheric perfusion related to carotid ligation. Some patients were sedated for MR imaging. However, given the small number of patients with abnormal arterial spin-labeling results who were sedated (4 of 25 total neonates), the exact impact is difficult to determine. Furthermore, the perfusion pattern in these neonates was quite variable. Of these 4 neonates, 2 had asymmetric perfusion (left side more pronounced than right), 1 had symmetrically increased perfusion, and 1 had symmetrically decreased perfusion.

Our findings of increased number and severity of injuries seen with VA ECMO are in accordance with prior literature. $^{22}$ It should be noted that those neonates who undergo VA ECMO generally demonstrate inherently increased disease severity compared with those on VV ECMO, necessitating both cardiovascular and respiratory support. We have shown that decreasing caliber of the distal right ICA is a common finding in those neonates who have undergone VA ECMO, likely as a result of altered vascular anatomy after carotid cannulation. It is uncertain why some patients treated with VA ECMO had no evidence of ICA narrowing. This finding may relate to collateralization of flow (such as external carotid to ICA collateral vascularization beyond the point of ligation).

Neuroimaging findings may prove useful in the prediction of neurodevelopmental outcomes in neonates who have undergone ECMO. Unfortunately, we were unable to collect robust longterm neurodevelopmental outcome data in this retrospective study, and therefore, we were not able to assess the relationship of reported imaging findings with functional deficits. Prior studies have shown that intracranial injury diagnosed on neuroimaging studies can predict future neurologic deficits in other high-risk neonatal patients, ${ }^{23}$ including patients treated with VA ECMO. ${ }^{7,24}$ However, a recent study questioned the prognostic value of MR imaging in neonatal patients treated with ECMO, though this study included a relatively small number of patients with outcome data. ${ }^{6}$ Future studies are needed in larger populations of neonatal ECMO survivors to establish functional correlates of specific MR imaging findings because this information can help guide family counseling and the direction of rehabilitative care.

Our study has several limitations. Our results have an inherent survivorship bias in that only those neonates who survived ECMO were included in our study. Therefore, it is more than likely that overall injury from ECMO is worse than what we have reported. However, it is this survivorship data that will be most useful for correlation with neurodevelopmental outcomes and subsequent use for counseling of families of ECMO survivors. Another limitation is the fact that the MR imaging protocols were not stan- 
dardized across our research population. The variability in the timing of MR imaging after ECMO decannulation precluded the ability to precisely time lesions to determine acuity and relationship to ECMO. This study spanned a 10 -year period, practically an eternity when it comes to the rapid pace of innovation in radiology. In keeping pace with such innovation, MR imaging protocols and sequence parameters were updated on a continuous basis. Although our cohort of patients was larger than that of most previous studies, our study remains limited by the relatively small overall sample size, especially in the evaluation of arterial spinlabeling and MR spectroscopy.

\section{CONCLUSIONS}

In a large contemporary cohort of neonatal patients treated with ECMO, MR imaging identified brain injury in nearly half of the patients after treatment, with increased sensitivity compared with sonography. The frontal and temporoparietal white matter are most commonly affected, without significant laterality. This pattern of injury is similar between VV ECMO and VA ECMO, though frequency of injury may be higher after VA ECMO. Future studies should strive to correlate post-ECMO injury with neurodevelopmental outcomes.

\section{ACKNOWLEDGMENTS}

The authors thank R.B. Govindan for his graphical assistance.

Disclosures: Michael A. Wien—RELATED: Grants/Grants Pending: Extracorporeal Life Support Organization*; Support for Travel to Meetings for the Study or Other Purposes: Extracorporeal Life Support Organization. * Matthew T. Whitehead—RELATED: Grants/ Grants Pending: Extracorporeal Life Support Organization*. Dorothy BulasUNRELATED: Consultancy: National Institutes of Health MOMS study. Michelande Ridore-RELATED: Grants/Grants Pending: ELSO, Comments: grant for "Characterization of cerebral injury by MRI in the modern era of neonatal ECMO."* An N. MassaroRELATED: Extracorporeal Life Support Organization. * * Money paid to the institution.

\section{REFERENCES}

1. Cengiz P, Seidel K, Rycus PT, et al. Central nervous system complications during pediatric extracorporeal life support: incidence and risk factors. Crit Care Med 2005;33:2817-24 CrossRef Medline

2. Teele SA, Salvin JW, Barrett CS, et al. The association of carotid artery cannulation and neurologic injury in pediatric patients supported with venoarterial extracorporeal membrane oxygenation*. Pediatr Crit Care Med 2014;15:355-61 CrossRef Medline

3. Oliver WC. Anticoagulation and coagulation management for ECMO. Semin Cardiothorac Vasc Anesth 2009;13:154-75 CrossRef Medline

4. van Heijst AF, de Mol AC, Ijsselstijn H. ECMO in neonates: neuroimaging findings and outcome. Semin Perinatol 2014;38:104-13 CrossRef Medline

5. Bulas DI, Taylor GA, O'Donnell RM, et al. Intracranial abnormalities in infants treated with extracorporeal membrane oxygenation: update on sonographic and CT findings. AJNR Am J Neuroradiol 1996;17:287-94 Medline

6. Rollins MD, Yoder BA, Moore KR, et al. Utility of neuroradiographic imaging in predicting outcomes after neonatal extracorporeal membrane oxygenation. J Pediatr Surg 2012;47:76-80 CrossRef Medline

7. Griffin MP, Minifee PK, Landry SH, et al. Neurodevelopmental outcome in neonates after extracorporeal membrane oxygenation: cranial magnetic resonance imaging and ultrasonography correlation. J Pediatr Surg 1992;27:33-35 CrossRef Medline

8. Liebeskind DS, Sanossian N, Sapo ML, et al. Cerebral microbleeds after use of extracorporeal membrane oxygenation in children. J Neuroimaging 2013;23:75-78 CrossRef Medline

9. Lago P, Rebsamen S, Clancy RR, et al. MRI, MRA, and neurodevelopmental outcome following neonatal ECMO. Pediatr Neurol 1995; 12:294-304 CrossRef Medline

10. Roelants-van Rijn AM, van der Grond J, de Vries LS, et al. Cerebral proton magnetic resonance spectroscopy of neonates after extracorporeal membrane oxygenation. Acta Paediatr 2001;90:1288-91 CrossRef Medline

11. Wiznitzer M, Masaryk TJ, Lewin J, et al. Parenchymal and vascular magnetic resonance imaging of the brain after extracorporeal membrane oxygenation. Am J Dis Child 1990;144:1323-26 CrossRef Medline

12. Parizel PM, Makkat S, Van Miert E, et al. Intracranial hemorrhage: principles of CT and MRI interpretation. Eur Radiol 2001;11: 1770-83 CrossRef Medline

13. Allen LM, Hasso AN, Handwerker J, et al. Sequence-specific MR imaging findings that are useful in dating ischemic stroke. Radiographics 2012;32:1285-97 CrossRef Medline

14. Bulas DI, Glass P, O'Donnell RM, et al. Neonates treated with ECMO: predictive value of early CT and US neuroimaging findings on short-term neurodevelopmental outcome. Radiology 1995;195: 407-12 CrossRef Medline

15. Raets MM, Dudink J, Ijsselstijn $\mathrm{H}$, et al. Brain injury associated with neonatal extracorporeal membrane oxygenation in the Netherlands: a nationwide evaluation spanning two decades. Pediatr Crit Care Med 2013;14:884-92 CrossRef Medline

16. Lazar EL, Abramson SJ, Weinstein S, et al. Neuroimaging of brain injury in neonates treated with extracorporeal membrane oxygenation: lessons learned from serial examinations. J Pediatr Surg 1994;29: 186-90; discussion 190-91 CrossRef Medline

17. Taylor GA, Walker LK. Intracranial venous system in newborns treated with extracorporeal membrane oxygenation: Doppler US evaluation after ligation of the right jugular vein. Radiology 1992; 183:453-56 CrossRef Medline

18. McLaughlin JF, Loeser JD, Roberts TS. Acquired hydrocephalus associated with superior vena cava syndrome in infants. Childs Nerv Syst 1997;13:59-63 CrossRef Medline

19. Groenendaal F, Veenhoven RH, van der Grond J, et al. Cerebral lactate and $\mathrm{N}$-acetyl-aspartate/choline ratios in asphyxiated full-term neonates demonstrated in vivo using proton magnetic resonance spectroscopy. Pediatr Res 1994;35:148-51 CrossRef Medline

20. Roelants-Van Rijn AM, van der Grond J, de Vries LS, et al. Value of (1)H-MRS using different echo times in neonates with cerebral hypoxia-ischemia. Pediatr Res 2001;49:356-62 CrossRef Medline

21. Deibler AR, Pollock JM, Kraft RA, et al. Arterial spin-labeling in routine clinical practice, part 3: hyperperfusion patterns. AJNR Am J Neuroradiol 2008;29:1428-35 CrossRef Medline

22. Maslach-Hubbard A, Bratton SL. Extracorporeal membrane oxygenation for pediatric respiratory failure: history, development and current status. World J Crit Care Med 2013;2:29-39 CrossRef Medline

23. Woodward LJ, Anderson PJ, Austin NC, et al. Neonatal MRI to predict neurodevelopmental outcomes in preterm infants. $N$ Engl J Med 2006;355:685-94 CrossRef Medline

24. Bulas D, Glass P. Neonatal ECMO: neuroimaging and neurodevelopmental outcome. Semin Perinatol 2005;29:58-65 CrossRef Medline 